\title{
Military Medical Research reviewer acknowledgement 2014-2015
}

Xiao-Bing Fu

\section{Contributing reviewers}

The editors of Military Medical Research would like to thank all our reviewers who have contributed to the journal in volume 1 (2014) and 2 (2015).

Maria das Graças Anguera

Brazil

Christian Bautista

United States of America

Eric Bernes

Switzerland

Zhao-xiang Bian

Hong Kong

Timothy Billiar

United States of America

Selami Çakmak

Turkey

Chuang Cai

China

Guangwen Cao

China

Wu-Chun Cao

China

Roberto Casale

Italy

Kevin Casey

Afghanistan

Theresa M. Casey

United States of America

Anne-Marie Chang

United States of America

\author{
Bohua Chen \\ China \\ Yao-Long Chen \\ China \\ Ning-Hai Cheng \\ China \\ Angela Chow \\ Singapore
}

Ahmet Yilmaz Coban

Turkey

Laura Coffey

Ireland

Le Cong

United States of America

Travis Craddock

United States of America

Robert Dahnke

Germany

Domenico De Berardis

Italy

Andrew Dwork

United States of America

Roberto Augusto Estrada Castañon Mexico

Christiane Kruse Fæste

Norway

\author{
Ibrahima Soce Fall \\ Senegal \\ Jie Fan \\ United States of America \\ Yi-Qun Fang \\ China
}

\section{Cheng Feng}

China

\section{Wolfgang Freund \\ Germany}

Carlo Fremd

Germany

\section{Xiao-Bing Fu}

China

Hui Gao

China

Xu-Bing Gao

China

Angelo Gemignani

Italy

Yan Geng

China

\section{Aayush Gupta}

India

Marios Hadjicharalambous

Cyprus 
John Harkness

Australia

Rod Hay

United Kingdom

Antonia Hildebrand
Germany

Hiroyuki Hirasawa

Japan

Yamashita Hiromasa

Japan

Lan Huang

China

Sha Huang

China

Sigurd Hystad

Norway

Jian-Xin Jiang

China

Ronald Kessler

United States of America

Jae-Seok Kim

South Korea

Young Wan Kim

South Korea

Luther C. Kloth

United States of America

Yankaskas Kurt

United States of America

Bas De Laat

Netherlands

Xi-Lan Lai

China

Li Li

China

Jing Li

China

Shao-hua Li

United States of America

You-Sheng Li

China

Thomas Liesegang

United States of America

Ji-Long Liu

United Kingdom
Wei Liu

China

Wei-Wei Liu

China

Xiao-rong Liu

China

Xin Luo

China

Gang Lv

China

Vinay Mahishale

India

Luis Massuca

Portugal

Ing How Moo

Singapore

Shawnda Morrison

Slovenia

Stephen Muza

United States Minor Outlying Islands

David Nelson

United States of America

Eisuke Ochi

Japan

John Park

United States of America

Didier Payen

France

Xuetao Pei

China

Rui-yun Peng

China

Yang Peng

United States of America

Sandeep Phatak

United States of America

Ivan Poon

Australia

Georgi Popivanov

Bulgaria

Larry Pruitt

United States of America

Xing-Shun Qi

China
Wei-Min Qu

China

Daniel G. Remick

United States of America

Samaneh Rouhi

Iran

Frank A. J. L. Scheer

United States of America

Alexa Smith-Osborne

United States of America

Mohan L. Sopori

United States of America

Michael Steketee

United States of America

Lei Su

China

Kentaro Sugano

Japan

Xin Sun

China

Zachary Taylor

United States of America

Stefania Tegli

Italy

Marcia Testa

United States of America

Gursimran Thandi

United Kingdom

James Tsai

United States of America

Jacob Turner

United States of America

Bhan Urvashi

United States of America

Timothy R. Varney

United States of America

Lakshmi Vas

India

Lee Vernon

Singapore

Michael Waller

Australia

De-yun Wang

Singapore 
Fu-Sheng Wang

China

Guo-qiang Wang

China

Hong-ying Wang

China

Jiang Huai Wang

Ireland

Kuang-Te Wang

Taiwan

Yan-Jiang Wang

China

Yang Wang

United States of America

Bryant Webber

United States of America

Rodney Willoughby

United States of America
Mee Lian Wong

Singapore

Michael Wünning

Germany

Xuan Xiao

China

Tai Xie

China

Noriko Yaekashiwa

Japan

Xiao Yang

China

Zheng-lin Yang

China

Yong-Ming Yao

China

Xue Yu

United States of America
Jing Yuan

China

Paul Zarogoulidis

Greece

Lian-yang Zhang

China

Min Zhao

United States of America

Fei-Hu Zhou

China

Qi-Quan Zhou

China

Jure Zupan

Slovenia 